\title{
ECG Acquired with Precordial Electrodes Placed on the Right Chest
}

National Cancer Institute

\section{Source}

National Cancer Institute. ECG Acquired with Precordial Electrodes Placed on the Right

Chest. NCl Thesaurus. Code C114175.

An electrocardiographic recording in which the precordial electrodes have been placed over the right chest and record a right sided ECG. (CDISC) 